Phys. Rev. Lett 96140401 (2006)

\title{
Bose-Einstein Condensation with Entangled Order Parameter
}

\author{
$\mathrm{Yu} \mathrm{Shi}^{1, *}$ and Qian $\mathrm{Niu}^{2}$ \\ ${ }^{1}$ Department Physics, Fudan University, Shanghai 200433, China \\ ${ }^{2}$ Department of Physics, University of Texas, Austin, TX 78712
}

\begin{abstract}
We propose a practically accessible non-mean-field ground state of Bose-Einstein condensation (BEC), which occurs in an interspecies two-particle entangled state, and is thus described by an entangled order parameter. A suitably defined entanglement entropy is used as the characterization of the non-mean-field nature, and is found to persist in a wide parameter regime. The interspecies entanglement leads to novel interference terms in the dynamical equations governing the single particle orbital wavefunctions. Experimental feasibility and several methods of probe are discussed. We urge the study of multi-channel scattering between different species of atoms.
\end{abstract}

PACS numbers: 03.67.Mn, 03.65.Ud

For a mixture of two distinct quantum many-particle systems, the usual approach is to associate an order parameter to each. For example, in a two-component BEC [1, 2, 3, 4], each component is separately described by a condensate wavefunction, although the two wavefunctions are classically coupled. Beyond this approach is BEC of spin-1 [5, 6, 7, 8] or spin- $\frac{1}{2}$ atoms [9, 10], but under consideration is one many-particle system in which all the atoms are indistinguishable and each atom can jump in between different spin states. In this Letter, we propose a peculiar mixture of two distinct species of atoms $a$ and $b$, which represents a novel situation different from both cases above. Each atom can flip between two pseudo spin states $\uparrow$ and $\downarrow$, but cannot transit between $a$ and $b$. The total occupation number of each species is conserved, but that of each spin state of each species is not conserved because of scattering between different species. It is shown that the ground state is BEC of inter-species pairs in a two-particle state which, as the global BEC order parameter, is quantum-entangled. As such, this work extends the notion of entanglement to order parameters of many-body systems, and represents a direction different from other works on entanglement in BEC [1]. In particular, the entanglement between the two species can serve as a characterization of the non-mean-field nature. The detection methods are discussed. We also derive the dynamical equations of the single particle orbital wavefunctions, each containing an interference term which is absent in BEC mixtures studied previously. As a condensate of nonlocal Bose pairs, our system is also related to the condensation of bound molecules and of paired fermions [12]. Experimentally, the two pseudo spin states can be implemented as two hyperfine $m_{F}$ states in a magnetic trap. The atoms can be constrained in the two pseudo spin states by exploiting the conservations of energy and of the total $m_{F}$ in a scattering. Because of the implemental feasibility as well as the persistence of the entanglement in a wide parameter regime, this BEC system represents a practically accessible non-mean-field ground state, in contrast with previous BEC models.

In ignorance of the depletion, the orbit of each atom, of species $i(i=a, b)$ and with pseudo $\operatorname{spin} \sigma$, is constrained in the single-particle ground state $\phi_{i \sigma}\left(\mathbf{r}_{i}\right)$. The Hamiltonian can be written as $\mathcal{H}=\sum_{\sigma} f_{i \sigma} N_{i \sigma}+\frac{1}{2} \sum_{\sigma \sigma^{\prime}} K_{\sigma \sigma^{\prime}}^{(i i)} N_{i \sigma} N_{i \sigma^{\prime}}+$ $\sum_{\sigma \sigma^{\prime}} K_{\sigma \sigma^{\prime}}^{(a b)} N_{a \sigma} N_{b \sigma^{\prime}}+\frac{K_{e}}{2}\left(a_{\uparrow}^{\dagger} a_{\downarrow} b_{\downarrow}^{\dagger} b_{\uparrow}+a_{\downarrow}^{\dagger} a_{\uparrow} b_{\uparrow}^{\dagger} b_{\downarrow}\right)$, where $a_{\sigma}$ and $b_{\sigma}$ are annihilation operators, $N_{a \sigma}=a_{\sigma}^{\dagger} a_{\sigma}$, $N_{b \sigma}=b_{\sigma}^{\dagger} b_{\sigma}, K_{\sigma \sigma}^{i j}$ 's are related to scattering lengths [13], $f_{i \sigma} \equiv \epsilon_{i \sigma}-K_{\sigma \sigma}^{(i i)} / 2$, where $\epsilon_{i \sigma}$ is the single particle energy. $\epsilon_{a \uparrow}-\epsilon_{a \downarrow}=\epsilon_{b \downarrow}-\epsilon_{b \uparrow} . N_{i}=N_{i \uparrow}+N_{i \downarrow}$ is a constant. The last term in $\mathcal{H}$ describes the spin-exchange scattering, which changes the occupation of each spin state of each species and causes interspecies entanglement. Define $\mathbf{S}_{a}=\sum_{\sigma, \sigma^{\prime}} a_{\sigma}^{\dagger} \mathbf{s}_{\sigma \sigma^{\prime}} a_{\sigma^{\prime}}$ and $\mathbf{S}_{b}=\sum_{\sigma, \sigma^{\prime}} b_{\sigma}^{\dagger} \mathbf{s}_{\sigma \sigma^{\prime}} b_{\sigma^{\prime}}$, where $\mathbf{s}$ is the spin- $\frac{1}{2}$ operator. The common eigenstate of $S_{a}^{2}$ and $S_{a z}$ is $\left|S_{a}, m_{a}\right\rangle=\left|\frac{N_{a}}{2}+m_{a}\right\rangle_{a \uparrow}\left|\frac{N_{a}}{2}-m_{a}\right\rangle_{a \downarrow}$. Thus we transform the Hamiltonian into that of two coupled big spins $S_{a}=N_{a} / 2$ and $S_{b}=N_{b} / 2$,

$$
\begin{aligned}
\frac{\mathcal{H}}{J_{z}}= & \frac{K_{e}}{J_{z}}\left(S_{a x} S_{b x}+S_{a y} S_{b y}\right)+S_{a z} S_{b z} \\
& +B_{a} S_{a z}+B_{b} S_{b z}+C_{a} S_{a z}^{2}+C_{b} S_{b z}^{2}+\frac{E_{0}}{J_{z}},
\end{aligned}
$$

where $J_{z}=K_{\uparrow \uparrow}^{(a b)}+K_{\downarrow \downarrow}^{(a b)}-K_{\uparrow \downarrow}^{(a b)}-K_{\downarrow \uparrow}^{(a b)}, B_{a} J_{z}=$ $f_{a \uparrow}-f_{a \downarrow}+N_{a}\left(K_{\uparrow \uparrow}^{(a a)}-K_{\downarrow \downarrow}^{(a a)}\right) / 2+N_{b}\left(K_{\uparrow \uparrow}^{(a b)}+K_{\uparrow \downarrow}^{(a b)}-\right.$ $\left.K_{\downarrow \uparrow}^{(a b)}-K_{\downarrow \downarrow}^{(a b)}\right) / 2, \quad B_{b} J_{z}=f_{b \uparrow}-f_{b \downarrow}+N_{b}\left(K_{\uparrow \uparrow}^{(b b)}-\right.$ $\left.K_{\downarrow \downarrow}^{(b b)}\right) / 2+N_{a}\left(K_{\uparrow \uparrow}^{(a b)}+K_{\downarrow \uparrow}^{(a b)}-K_{\uparrow \downarrow}^{(a b)}-K_{\downarrow \downarrow}^{(a b)}\right) / 2, C_{a} J_{z}=$ $\left(K_{\uparrow \uparrow}^{(a a)}+K_{\downarrow \downarrow}^{(a a)}-K_{\uparrow \downarrow}^{(a a)}-K_{\downarrow \uparrow}^{(a a)}\right) / 2, C_{b} J_{z}=\left(K_{\uparrow \uparrow}^{(b b)}+\right.$ $\left.K_{\downarrow \downarrow}^{(b b)}-K_{\uparrow \downarrow}^{(b b)}-K_{\downarrow \uparrow}^{(b b)}\right) / 2 . \quad E_{0}=\sum_{i}\left(\sum_{\sigma} f_{i \sigma}\right) N_{i}+$ $(1 / 8) \sum_{i}\left(\sum_{\sigma \sigma^{\prime}} K_{\sigma \sigma^{\prime}}^{(i)}\right) N_{i}^{2}+(1 / 4)\left(\sum_{\sigma \sigma^{\prime}} K_{\sigma \sigma^{\prime}}^{(a b)}\right) N_{a} N_{b}$ is a constant and is neglected henceforth.

$\mathcal{H}$ conserves the total $z$-component $\operatorname{spin} S_{z}=\left(N_{a \uparrow}-\right.$ $\left.N_{a \downarrow}+N_{b \uparrow}-N_{b \downarrow}\right) / 2$. The total spin is $S=S_{a}-$ $S_{b}, \cdots, S_{a}+S_{b}$, letting $N_{a} \geq N_{b}$. For a given $S_{z}$, any eigenstate of $\mathcal{H}$ can be written as $|\Psi\rangle=$ $\sum_{m} g(m)\left|S_{a}, m\right\rangle\left|S_{b}, S_{z}-m\right\rangle$, with the summation range 
$\max \left(-S_{a}, S_{z}-S_{b}\right) \leq m \leq \min \left(S_{a}, S_{z}+S_{b}\right)$.

At the isotropic point, i.e. if $K_{e}=J_{z}$ while $B_{a}=$ $B_{b}=C_{a}=C_{b}=0$, the Hamiltonian is simplified as $\mathcal{H}=J_{z} \mathbf{S}_{a} \cdot \mathbf{S}_{b}$, whose eigenstates are nothing but $\left|S, S_{z}\right\rangle$, for which $g(m)$ is the Clebsch-Gordon coefficient $\left\langle S_{a}, m, S_{b}, S_{z}-m \mid S, S_{z}\right\rangle$. The eigenvalues are degenerate for different values of $S_{z}$. There are $N_{a}-N_{b}+1$ degenerate ground states $\left|G_{S_{z}}\right\rangle=\left|S_{a}-S_{b}, S_{z}\right\rangle$, which are found to be exactly

$$
\left|G_{S_{z}}\right\rangle=A\left(a_{\uparrow}^{\dagger}\right)^{n_{\uparrow}}\left(a_{\downarrow}^{\dagger}\right)^{n_{\downarrow}}\left(a_{\uparrow}^{\dagger} b_{\downarrow}^{\dagger}-a_{\downarrow}^{\dagger} b_{\uparrow}^{\dagger}\right)^{N_{b}}|0\rangle,
$$

where $n_{\uparrow}=N_{a} / 2-N_{b} / 2+S_{z}, n_{\downarrow}=N_{a} / 2-N_{b} / 2-S_{z}$, $A=\left[\left(N_{a}-N_{b}+1\right) ! /\left(N_{a}+1\right) ! N_{b} !\left(N_{a} / 2-N_{b} / 2+\right.\right.$ $\left.\left.S_{z}\right) !\left(N_{a} / 2-N_{b} / 2-S_{z}\right) !\right]^{1 / 2}$ is the normalization factor. Particularly, for $N_{a}=N_{b}=N$, the ground state is uniquely $\left|G_{0}\right\rangle=(\sqrt{N+1} N !)^{-1}\left(a_{\uparrow}^{\dagger} b_{\downarrow}^{\dagger}-a_{\downarrow}^{\dagger} b_{\uparrow}^{\dagger}\right)^{N}|0\rangle$.

The non-mean-field nature of these ground states can be well characterized by the entanglement between the two species. Rewrite $\left|G_{0}\right\rangle$ as $(\sqrt{N+1})^{-1} \sum_{m=0}^{N}(-1)^{N-m}|m\rangle_{a \uparrow}|N-m\rangle_{a \downarrow}|N-m\rangle_{b \uparrow}|m\rangle_{b \downarrow}$. The entanglement between the occupation of each one of the four single particle states and the rest of the system can be quantified as the von Neumann entropy of its reduced density matrix [14], which is $\log _{N+1}(N+1)=1$, where we set the logarithmic base to be $N+1$ because for each single particle basis state, the occupation number is $(N+1)$-valued. In a sense $\left|G_{0}\right\rangle$ is maximally entangled, due to equal superposition of the $N+1$ quart-orthogonal states. As each species comprises two spin states, it can be seen that the entropy of the entanglement between the total $a$ species and the total $b$ species is given by the same entropy. For the general eigenstate $|\Psi\rangle$ above, one can also write it in terms of occupation numbers in four single particle states, and obtain the entanglement entropy as $-\sum_{m} g^{2}(m) \log _{N_{b}+1} g^{2}(m)$, which must be less than 1 if $|\Psi\rangle \neq\left|G_{0}\right\rangle$, as $g(m)^{2}$ 's are not all equal for different $m$ 's.

$\left|G_{S_{z}}\right\rangle$ is a condensation of interspecies pairs in the same two-particle entangled state $\phi\left(\mathbf{r}_{a}, \mathbf{r}_{b}\right) \equiv \frac{1}{\sqrt{2}}\left[\phi_{a \uparrow}\left(\mathbf{r}_{a}\right)|\quad \uparrow\rangle_{a} \phi_{b \downarrow}\left(\mathbf{r}_{b}\right)|\quad \downarrow\rangle_{b} \quad-\right.$ $\left.\phi_{a \downarrow}\left(\mathbf{r}_{a}\right)|\quad \downarrow\rangle_{a} \phi_{b \uparrow}\left(\mathbf{r}_{b}\right)|\uparrow\rangle_{b}\right]$, plus the extra $a$-atoms. This can be seen by noting $\left(a_{\uparrow}^{\dagger} b_{\downarrow}^{\dagger}-a_{\downarrow}^{\dagger} b_{\uparrow}^{\dagger}\right)^{N_{b}}=$ $\left[\sqrt{2} \int d^{3} r_{a} d^{3} r_{b} \psi_{a}^{\dagger}\left(\mathbf{r}_{a}\right) \psi_{b}^{\dagger}\left(\mathbf{r}_{b}\right) \phi\left(\mathbf{r}_{a}, \mathbf{r}_{b}\right)\right]^{N_{b}}, \quad$ where $\psi_{a}(\mathbf{r})=\sum_{\sigma} a_{\sigma} \phi_{a \sigma}\left(\mathbf{r}_{a}\right)|\sigma\rangle_{a}, \psi_{b}(\mathbf{r})=\sum_{\sigma} b_{\sigma} \phi_{b \sigma}\left(\mathbf{r}_{b}\right)|\sigma\rangle_{b}$. Thus the BEC order parameter, given by $\phi\left(\mathbf{r}_{a}, \mathbf{r}_{b}\right)$, is quantum-entangled [15]. Like Cooper pairing, the formation of the entangled two-particle state lowers the energy, as can be seen by considering one $a$-atom and one $b$-atom, with Hamiltonian $h\left(\mathbf{r}_{a}\right)+h\left(\mathbf{r}_{b}\right)+U_{1}\left(\mathbf{r}_{a}-\mathbf{r}_{b}\right)+U_{2}\left(\mathbf{r}_{a}-\mathbf{r}_{b}\right)(|\uparrow \downarrow\rangle\langle\uparrow \downarrow|+| \downarrow \uparrow\rangle\langle\downarrow \uparrow$ $\mid)$, where $U_{2}>0$. Compared with the separable state $\phi_{a}\left(\mathbf{r}_{a}\right) \phi_{b}\left(\mathbf{r}_{b}\right)|\sigma\rangle\left|\sigma^{\prime}\right\rangle$, the entangled two-particle state $\phi_{a}\left(\mathbf{r}_{a}\right) \phi_{b}\left(\mathbf{r}_{b}\right)(|\uparrow\rangle|\downarrow\rangle-|\downarrow\rangle|\uparrow\rangle) / \sqrt{2}$ lowers the energy by $\int U_{2}^{(a b)}\left(\mathbf{r}_{a}-\mathbf{r}_{b}\right)\left|\phi_{a}\left(\mathbf{r}_{a}\right) \phi_{b}\left(\mathbf{r}_{b}\right)\right|^{2}$. For such energetic reason, our BEC occurs globally in the interspecies entangled pair state $\phi\left(\mathbf{r}_{a}, \mathbf{r}_{b}\right)$, rather than separately in the two species.

This entanglement can be experimentally probed by several means. First, because of entanglement, the particle number of each spin state of each species is subject to strong fluctuation. For $\left|G_{0}\right\rangle$, $\left\langle N_{i \sigma}\right\rangle=N / 2,\left\langle N_{a \sigma}^{2}\right\rangle-\left\langle N_{i \sigma}\right\rangle^{2}=N(N+2) / 12$, and thus $\sqrt{\left\langle N_{a \sigma}^{2}\right\rangle-\left\langle N_{a \sigma}\right\rangle^{2}} /\left\langle N_{a \sigma}\right\rangle \approx 1 / \sqrt{3}$. Note that the quantum mechanical average can only be obtained through several runs of the measurement. Nevertheless, as the density is a self-averaging quantity [16], one can study, in a single image, the fluctuation of the density $\rho_{i \sigma}\left(\mathbf{r}_{i}\right)=N_{i \sigma}\left|\phi_{i \sigma}\left(\mathbf{r}_{i}\right)\right|^{2}$. Interestingly, $\sqrt{\left\langle\rho_{i \sigma}\left(\mathbf{r}_{i}\right)^{2}\right\rangle-\left\langle\rho_{i \sigma}\left(\mathbf{r}_{i}\right)\right\rangle^{2}} /\left\langle\rho_{i \sigma}\left(\mathbf{r}_{i}\right)\right\rangle=$ $\sqrt{\left\langle N_{i \sigma}^{2}\right\rangle-\left\langle N_{i \sigma}\right\rangle^{2}} /\left\langle N_{i \sigma}\right\rangle$.

Secondly, this entanglement is also indicated by nonvanishing of the connected correlations between particle numbers of the two species, $C_{\sigma, \sigma^{\prime}} \equiv$ $\left\langle N_{a \sigma} N_{b \sigma^{\prime}}\right\rangle-\left\langle N_{a \sigma}\right\rangle\left\langle N_{b \sigma^{\prime}}\right\rangle$. For $\left|G_{0}\right\rangle, \quad C_{\sigma, \sigma}=$ $-N(N+2) / 12, \quad C_{\sigma, \bar{\sigma}}=N(N+2) / 12$, where $\bar{\sigma} \neq \sigma$. Again, these connected correlations can be measured through the corresponding quantities of the densities, $g\left(\mathbf{r}_{a}, \sigma ; \mathbf{r}_{b}, \sigma^{\prime}\right) \equiv\left\langle\rho_{a \sigma}\left(\mathbf{r}_{a}\right) \rho_{b \sigma^{\prime}}\left(\mathbf{r}_{b}\right)\right\rangle-$ $\left\langle\rho_{a \sigma}\left(\mathbf{r}_{a}\right)\right\rangle\left\langle\rho_{b \sigma^{\prime}}\left(\mathbf{r}_{b}\right)\right\rangle=C_{\sigma, \sigma^{\prime}}\left|\phi_{a \sigma}\left(\mathbf{r}_{a}\right) \phi_{b \sigma}\left(\mathbf{r}_{b}\right)\right|^{2}, \quad$ and $g\left(\mathbf{r}_{a}, \sigma ; \mathbf{r}_{b}, \sigma^{\prime}\right) /\left\langle\rho_{a \sigma}\left(\mathbf{r}_{a}\right)\right\rangle\left\langle\rho_{b \sigma^{\prime}}\left(\mathbf{r}_{b}\right)\right\rangle=C_{\sigma, \sigma^{\prime}} /\left\langle N_{a \sigma}\right\rangle\left\langle N_{b \sigma^{\prime}}\right\rangle$.

These density fluctuations and correlations are those in the original condensate, so it is not necessary to switch off the trap to let the condensate freely expand. Nevertheless, one can do so in order to obtain a larger image, which equally contains information about the entanglement. This is because the free propagator does not affect the entanglement. Moreover, the density fluctuations and correlations in the expanded gas are proportional to corresponding quantities in momentum space [16], which are related to those of the particle numbers in a way similar to that in the coordinate space, as described above.

Thirdly, this entanglement can also be probed by detecting atoms which leave the trap. Consider two-atom measurements in which the joint probability is obtained. In measuring the spin state of an $a$-atom, the probability of obtaining $\sigma$ is $P_{i \sigma}=$ $\left\langle a_{\sigma}^{\dagger} a_{\sigma}\right\rangle / \sum_{\sigma^{\prime}}\left\langle a_{\sigma^{\prime}}^{\dagger} a_{\sigma^{\prime}}\right\rangle$ [17. For a joint measurement of the spins of an $a$-atom and a $b$-atom, the probability of obtaining $\sigma$ for the $a$-atom while $\sigma^{\prime}$ for the $b$-atom is $P_{\sigma, \sigma^{\prime}}=\left\langle b_{\sigma^{\prime}}^{\dagger} a_{\sigma}^{\dagger} a_{\sigma} b_{\sigma^{\prime}}\right\rangle / \sum_{\sigma_{a}, \sigma_{b}}\left\langle b_{\sigma_{b}}^{\dagger} a_{\sigma_{a}}^{\dagger} a_{\sigma_{a}} b_{\sigma_{b}}\right\rangle$. A nonentangled, or mean-field type, state is in the form of $\left(\sqrt{N_{1} ! N_{2} ! N_{3} ! N_{4} !}\right)^{-1} a_{\hat{\mathbf{n}}}^{\dagger N_{1}} a_{-\hat{\mathbf{n}}}^{\dagger} b_{\hat{\mathbf{m}}}^{N_{2}} b_{-\hat{\mathbf{m}}}^{N_{3}}|0\rangle$, where $\hat{\mathbf{n}}$ and $\hat{\mathbf{m}}$ are two arbitrary "directions". Hence for arbitrary chosen $\sigma_{a}$ and $\sigma_{b}, P_{\sigma_{a}, \sigma_{b}}=P_{\sigma_{a}} P_{\sigma_{b}}$. In contrast, for an entangled BEC considered here, $P_{\sigma_{a}, \sigma_{b}} \neq P_{\sigma_{a}} P_{\sigma_{b}}$. Indeed, for $\left|G_{0}\right\rangle, P_{\sigma_{a}}=P_{\sigma_{b}}=1 / 2$, but $P_{\uparrow \downarrow}=P_{\downarrow \uparrow}=$ $(2 N+1) / 6 N$ while $P_{\uparrow \uparrow}=P_{\downarrow \downarrow}=(N-1) / 6 N$.

Finally, this entanglement has feedback effects on the single particle orbits. We can derive the equations governing the orbital wavefunctions, as the coun- 


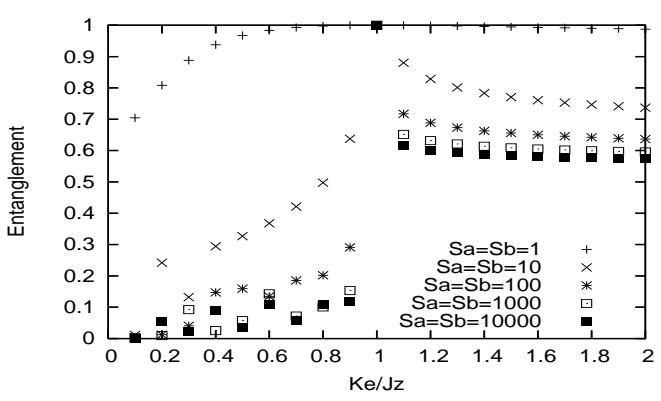

FIG. 1: The entanglement as a function of $K_{e} / J_{z}$, with $B_{a}=B_{b}=C_{a}=C_{b}=0, S_{z}=0$. The entanglement measure used both here and in the next three figures is the von Neumann entropy of the reduced density matrix of either of the two species, calculated by considering the occupation entanglement [14].

terparts of the Gross-Pitaevskii equation, using the method of Ashhab and Leggett [10]. The minimization of the expectation value of $\mathcal{H}$ in the ground state $\left|G_{0}\right\rangle$ gives rise to $\left\{-\frac{\hbar^{2}}{2 m_{a}} \nabla^{2}+U_{a \sigma}(\mathbf{r})+[N(N-\right.$ 1) $/ 3] g_{\sigma \sigma}^{(a)}\left|\phi_{a \sigma}(\mathbf{r})\right|^{2}+[N(N-1) / 6] g_{\uparrow \downarrow}^{(a a)}\left|\phi_{a \bar{\sigma}}(\mathbf{r})\right|^{2}+[N(N-$ 1) $\left./ 6] g_{\sigma \sigma}^{(a b)}\left|\phi_{b \sigma}(\mathbf{r})\right|^{2}+[N(2 N+1) / 6] g_{\sigma \bar{\sigma}}^{(a b)}\left|\phi_{b \bar{\sigma}}(\mathbf{r})\right|^{2}\right\} \phi_{a \sigma}(\mathbf{r})-$ $[N(N+2) / 12] g_{e} \phi_{b \bar{\sigma}}^{*}(\mathbf{r}) \phi_{b \sigma}(\mathbf{r}) \phi_{a \bar{\sigma}}(\mathbf{r})=\mu_{a \sigma} \phi_{a \sigma}(\mathbf{r})$ and the likes for the other three wavefunctions, where $g_{\sigma \sigma^{\prime}}^{(i j)}$ and $g_{e}$ are the coefficients in front of the integrals in $K_{\sigma \sigma^{\prime}}^{(i j)}$ and $K_{e}$ [13]. The term proportional to $g_{e}$ is an interference term, which persists even in the Thomas-Fermi limit. It is absent in previous models and may lead to novel physical properties.

We now study how this entanglement survives the coupling anisotropy $J_{z} \neq K_{e}$ and the nonvanishing of $B_{a}$, $B_{b}, C_{a}$ and $C_{b}$. We use the Lanczos method to numerically determine the ground states for different parameter values. First we study how the entanglement varies with $K_{e} / J_{z}$, setting $B_{a}=B_{b}=C_{a}=C_{b}=0$. As shown in Figs. 1 and 2, the entanglement is maximized when $K_{e}=J_{z}$, and is maximized at $S_{z}=0$. This maximum is 1 if $S_{a}=S_{b}$, and is less than 1 otherwise. Roughly speaking, the larger the spins, the sharper the deviation of the entanglement from the maximum, but the details depend on all of $S_{a}, S_{b}$ and $S_{z}$. The decrease for $K_{e} / J_{z}>1$ is much slower than the increase for $K_{e} / J_{z}<1$.

Under typical values $S_{a}=12000, S_{b}=10000, S_{z}=$ $1000, K_{e} / J_{z}=1.2$, we study how the entanglement varies with $C_{a}$ and $C_{b}$, while $B_{a}=B_{b}=0$. Because $J_{z}, C_{a} J_{z}$ and $C_{b} J_{z}$ are about of the same order of magnitude of $K_{\sigma \sigma^{\prime}}^{(i j)}$ 's, we consider the range $-2 \leq C_{a} \leq 2$ and $-2 \leq$ $C_{b} \leq 2$. As shown in Fig. 3, the change of entanglement from the case of $C_{a}=C_{b}=0$, in which the entanglement is 0.6 , is quite limited.

Then we typically choose $C_{a}=0.2$ and $C_{b}=0.4$, and study the variation of the entanglement with $B_{a}$ and $B_{b}$. As $B_{a}$ and $B_{b}$ can be as large as the order of magni-

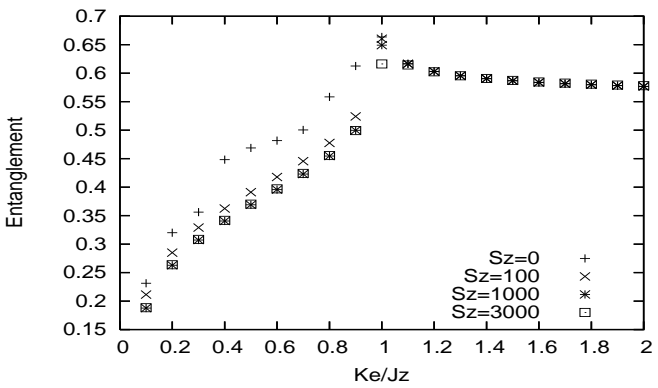

FIG. 2: The entanglement as a function of $K_{e} / J_{z}$, with $B_{a}=$ $B_{b}=C_{a}=C_{b}=0, S_{a}=12000, S_{b}=10000$.

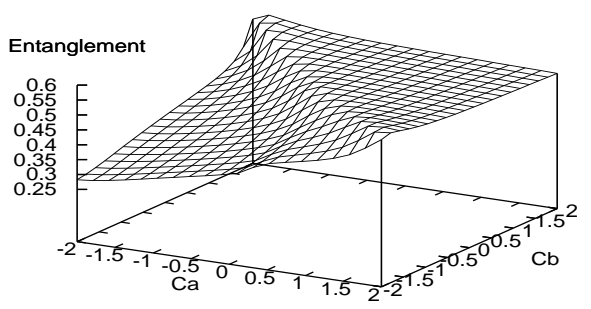

FIG. 3: The entanglement as a function of $C_{a}$ and $C_{b}$, with $S_{a}=12000, S_{b}=10000, S_{z}=1000, K_{e} / J_{z}=1.2, B_{a}=$ $B_{b}=0$.

tude of $N_{a}$ and $N_{b}$ or even larger, we consider the range $-10000 \leq B_{a} \leq 10000$ and $-10000 \leq B_{b} \leq 10000$. Fig. 4 indicates that in such a parameter range, the variation of entanglement, with the maximum 0.57 , is still quite limited.

These results show that BEC with an inter-species entangled order parameter, as a highly non-mean-field ground state, can occur in a quite large parameter regime. The reason why the entanglement is not easily destroyed by anisotropy and $C_{a}, C_{b}, B_{a}$ and $B_{b}$ is that the entanglement is caused by the exchange interaction. This leads to more relaxed condition for the persistence of entanglement than for the energetic pertur-

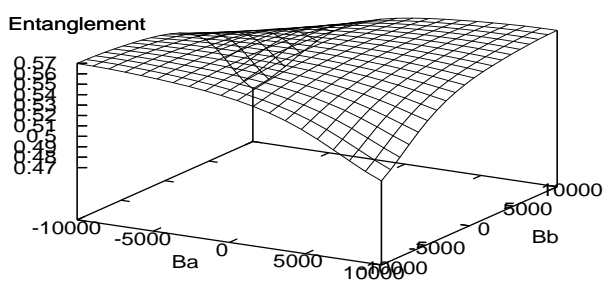

FIG. 4: The entanglement as a function of $B_{a}$ and $B_{b}$, with $S_{a}=12000, S_{b}=10000, S_{z}=1000, K_{e} / J_{z}=1.2, C_{a}=0.2$, $C_{b}=0.4$. 
bation. Moreover, around $C_{a} \approx-C_{b}$, there is a canceling effect in the additional term in the Hamiltonian, $C_{a} S_{a z}^{2}+C_{b} S_{b z}^{2}=\left(C_{a}+C_{b}\right) S_{a z}^{2}-2 C_{b} S_{z} S_{b z}+C_{b} S_{z}^{2}$. Similarly, around $B_{a} \approx B_{b}$, there is a canceling effect in $B_{a} S_{a z}+B_{b} S_{b z}=\left(B_{a}-B_{b}\right) S_{a z}+B_{b z} S_{z}$. The canceling effect further reduces the change of entanglement, as exhibited in the Figs. 3 and 4.

It is important that the non-mean-field ground state, with inter-species entanglement, can be accessed in experiments. A crucial issue in experimentally accessing the non-mean-field ground state is the energy gap. At the isotropic point of our Hamiltonian, in analogy with BEC of spin- 1 bosons, the gap is of the order of $\sim 1 / V$, thus the ground state is very difficult to be reached in practice [ $]$. This is spontaneous symmetry breaking. However, it can be estimated that when $B_{a}-B_{b}$ is of the order of $\pm N$ or $C_{a}+C_{b}$ is of the order of -1 , the energy gap is of the order of $N / V$, which is finite in the thermodynamic limit. On the other hand, we have seen above that in this parameter regime, as far as $K_{e}$ is larger than or not much smaller than $J_{z}$, the entanglement is significantly nonvanishing. Even if $B_{a}, B_{b}, C_{a}$ and $C_{b}$ are not so large, the finite energy gap can still be opened up when $J_{z} \gg K_{e}$. But then the entanglement tends to vanish. One may resort to adiabatic switching, which might be realized by using Feshbach resonance. One starts with the gapped case $J_{z} \gg K_{e}$. By adiabatically tuning $K_{e}$ towards $K_{e} \approx J_{z}$, the ground state evolves into a highly entangled one, though with vanishing gap.

The hyperfine-Zeeman energy levels depend only on $F$ and $m_{F}$, not on the species [8]. Therefore we can simply represent the same pseudo spin state of the two species by the same actual hyperfine state. To exactly realize our model, only forward and exchange scattering channels are needed, thus the scattering into other hyperfine states needs to be suppressed. A choice is $|\uparrow\rangle=\mid F=2, m_{F}=$ $2\rangle,|\downarrow\rangle=\left|F=2, m_{F}=1\right\rangle$. One may also use $|\uparrow\rangle=$ $\left|F=2, m_{F}=2\right\rangle,|\downarrow\rangle=\left|F=1, m_{F}=1\right\rangle$, or use $|\uparrow\rangle=$ $\left|F=2, m_{F}=-1\right\rangle,|\downarrow\rangle=\left|F=1, m_{F}=-1\right\rangle$, which are the spin states in the well-known two-component ${ }^{87} R b$ BECs 2]. In these previous experiments, because the particle number in each spin state is conserved, the BECs are equivalent to a mixture of two species, each in a fixed spin state, which was implemented in ${ }^{41} K$ and ${ }^{87} R b$, both in $\left|F=2, m_{F}=2\right\rangle$ [4]. These experimental experiences can exploited to realize our model. For the latter two choices of spin states, as the energy difference between the two spin states with different $F$ 's is very large, $B_{a}$ and $B_{b}$ can be of the order of $N$ or larger. Likely this can produce the gap. Lack of data on interspecies scattering prohibits more detailed realistic discussions here.

To summarize, we have proposed a practically accessible non-mean-field BEC, which is a condensation of interspecies spin-entangled pairs and can be characterized by a suitably defined entanglement entropy, which is maximized in the ground state at the isotropic limit, but remains significant in a wide parameter regime. We have also derived the dynamical equations of the single particle orbital wavefunctions, where there are novel interference terms. This non-mean-field ground state can even be gapped. We have discussed the experimental feasibility and put forward several means of experimental detection.

Y.S. is very grateful to Prof. Tony Leggett for precious advice, and to Fei Ye for generous help in numerics. We also thank Fei Zhou, Li You, Michael Stone, Biao Wu and Tao Li for useful discussions.

* Corresponding author.

Electronic address: yushi@fudan.edu.cn

[1] T. L. Ho and V. B. Shenoy, Phy. Rev. Lett. 77, 3276 (1996).

[2] C. J. Myatt, et al., Phy. Rev. Lett. 78, 586 (1997). D. S. Hall et al., Phy. Rev. Lett. 81, 1539 (1998). D. S. Hall et al., Phy. Rev. Lett. 81, 1543 (1998).

[3] I. Bloch et al., Phys. Rev. A 64, 021402(R) (2001). J. P. Burke et al. Phy. Rev. Lett. 80, 2097 (1998).

[4] G. Modugno et al. Phy. Rev. Lett. 89, 190404 (2002); G. Ferrari et al. Phy. Rev. Lett. 89, 053202 (2002).

[5] T. L. Ho, Phy. Rev. Lett. 81, 742 (1998); T. Ohmi and K. Machida, J. Phys. Soc. Jpn, 67, 1822 (1998); C. K. Law, H. Pu and N. P. Bigelow, Phy. Rev. Lett. 81, 5257 (1998); T. L. Ho and S. K. Yip, Phy. Rev. Lett. 84, 4031(2000).

[6] F. Zhou, Phy. Rev. Lett. 87, 080401 (2001).

[7] J. Stenger et al., Nature 396, 345 (1998); M. S. Chang et al., Nature Phys. 1, 111 (2005).

[8] A. J. Leggett, Rev. Mod. Phys. 73, 307 (2001).

[9] A. B. Kuklov and B. V. Svistunov, Phy. Rev. Lett. 89, 170403 (2002).

[10] S. Ashhab and A. J. Leggett, Phys. Rev. A 68, 063612 (2003)

[11] H. Pu and P. Meystre, Phys. Rev. Lett. 85, 3987 (2000); L. M. Duan et al., Phys. Rev. Lett. 85, 3991 (2000); A. Sorensen et al., Nature 409, 63 (2001); K. Helmerson and L. You, Phys. Rev. Lett. 87, 170402 (2001); J. M. Vogels, K. Xu and W. Ketterle, Phys. Rev. Lett. 89, 020401 (2002); B. Deb and G. S. Agarwal, Phys. Rev. A 65, 063618 (2002); A. P. Hines, R. H. McKenzie and G. J. Milburn, Phys. Rev. A 67, 013609 (2003); M. Jack and M. Yamashita, Phys. Rev. A 71, 033619 (2005).

[12] E. A. Donley et al., Nature 417, 529 (2002); M. Greiner et al., Nature 426, 537 (2003); S. Jochim et al., Science 302, 2101 (2003); M. W. Zwierlein et al., Phys. Rev. Lett. 91, 250401 (2003). C. A. Regal et al., Phys. Rev. Lett. 92, 040403 (2004); M. Bartenstein et al., Phys. Rev. Lett. 92, 120401 (2004); M. W. Zwierlein et al., Phys. Rev. Lett. 92, 120403 (2004); J. Kinast et al., Phys. Rev. Lett. 92, 150402 (2004); T. Bourdel et al., Phys. Rev. Lett. 93, 050401 (2004).

[13] For the scattering in which an $i$-atom flips from $\sigma_{4}$ to $\sigma_{1}$ while an $j$-atom flips from $\sigma_{3}$ to $\sigma_{2}$, with scattering length $\xi_{\sigma_{1} \sigma_{2} \sigma_{3} \sigma_{4}}^{(i j)}$ and effective mass $\mu_{i j}=m_{i} m_{j} /\left(m_{i}+m_{j}\right)$, define $K_{\sigma_{1} \sigma_{2} \sigma_{3} \sigma_{4}}^{(i j)} \equiv$ $\left(2 \pi \hbar^{2} \xi_{\sigma_{1} \sigma_{2} \sigma_{3} \sigma_{4}}^{(i j)} / \mu_{i j}\right) \int \phi_{i \sigma_{1}}^{*}(\mathbf{r}) \phi_{j \sigma_{2}}^{*}(\mathbf{r}) \phi_{j \sigma_{3}}(\mathbf{r}) \phi_{i \sigma_{4}}(\mathbf{r}) d^{3} r$. $K_{\sigma \sigma}^{(i i)} \equiv K_{\sigma \sigma \sigma \sigma}^{(i i)}, K_{\sigma \bar{\sigma}}^{(i i)} \equiv 2 K_{\sigma \bar{\sigma} \bar{\sigma} \sigma}^{(i i)}=2 K_{\sigma \bar{\sigma} \sigma \bar{\sigma}}^{(i i)}$ for $\sigma \neq \bar{\sigma}$, 
$K_{\sigma \sigma^{\prime}}^{(a b)} \equiv K_{\sigma \sigma^{\prime} \sigma^{\prime} \sigma}^{(a b)}, K_{e} \equiv 2 K_{\uparrow \downarrow \uparrow \downarrow}^{(a b)}=2 K_{\downarrow \uparrow \downarrow \uparrow}^{(a b)}$.

[14] See, e.g., Y. Shi, J. Phys. A 37, 6807 (2004).

[15] Y. Shi, Int. J. Mod. Phys. B 15, 3007 (2001).

[16] E. Altman, E. Demler and M. D. Lukin, Phys. Rev. A
70, 013603 (2004).

[17] S. Ashhab and A. J. Leggett, Phys. Rev. A 65, 023604 (2002). 